\title{
Stacking structures of dry phospholipid films on a solid substrate
}

\section{$\operatorname{AUTHOR}(S)$ :}

Hishida, M.; Seto, H.; Kaewsaiha, P.; Matsuoka, H.; Yoshikawa, $\mathrm{K}$.

\section{CITATION:}

Hishida, M....[et al]. Stacking structures of dry phospholipid films on a solid substrate. Colloids and Surfaces 2006, 284-285: 444-447

\section{ISSUE DATE:}

2006-08

URL:

http://hdl.handle.net/2433/49169

RIGHT:

Copyright (C) 2006 Elsevier B.V. All rights reserved. 


\title{
Stacking structures of dry phospholipid films on a solid substrate
}

\author{
M. Hishida ${ }^{\text {a }}$, H. Seto ${ }^{\text {a,* }}$, P. Kaewsaiha ${ }^{\text {b }}$, H. Matsuoka ${ }^{\text {, }}$, \\ K. Yoshikawa ${ }^{a}$ \\ ${ }^{a}$ Department of Physics, Kyoto University, Kyoto 606-8502, Japan \\ ${ }^{\mathrm{b}}$ Department of Polymer Chemistry, Kyoto University, Kyoto 615-8510, Japan
}

\begin{abstract}
Stacking structures and surface morphologies of dry phospholipid films on a solid substrate were investigated by X-ray Reflectivity and Atomic Force Microscopy. A DOPC (1,2-dioleoyl-sn-glycero-3-phosphocholine) film in the liquid crystalline phase stacked regularly and formed a terrace-like morphology, while a DPPC $(1,2-$ dipalmitoyl-sn-glycero-3-phosphocholine) film in the gel phase showed a rough morphology with random layering. This difference in the stacking structure is discussed in relation to the ability of formation of phospholipid giant vesicles by the natural swelling method (M. Hishida, H. Seto, K. Yoshikawa, Chem. Phys. Lett. 411 (2005) 267).
\end{abstract}

Key words: Phospholipid, Dry film, Morphology, X-ray reflectivity, Atomic Force Microscopy

\section{Introduction}

Phospholipid, which is the main constituent of the cell membrane, spontaneously forms micelles or vesicles in water due to its amphiphilic property. Among these, phospholipid giant vesicles (GV) ranging from 1-100 $\mu \mathrm{m}$ in diameter have been actively investigated as a model system of living cells [1-3]. Various methods have been developed for the effective preparation of GV, such

* Corresponding author. FAX: +81 757533749

Email address: st@scphys.kyoto-u.ac.jp (H. Seto). 
as the electro-formation method and the solvent-evaporation method [5-10]. However, the physical or chemical treatments involved in most of these methods are unsuitable for their use as a model cell system. On the other hand, the natural swelling method, in which GVs are effectively obtained by swelling dry phospholipid film without vortex, serves ideal model cell system due to the lack of any harmful effects during preparation [4,5]. Although the natural swelling method has been known for a long time, the mechanism of GV formation in this method is not yet fully understood. The experimental procedure still depends on technical skill without a solid physico-chemical background.

Several authors have investigated the mechanism of $\mathrm{GV}$ formation in the natural swelling method. Direct observations of the hydration process by phasecontrast microscopy have shown that the myelin figures grow in the form of tubular fibrils when dry phospholipid films are hydrated [5,12-14]. However, in these experiments the phospholipid film filled in between two parallel glass coverslips and the film was hydrated by a small amount of water. These conditions are far from those in the natural swelling method for the preparation of $\mathrm{GV}$, in which a small spot of dry film on a substrate is hydrated by large excess water. Lasic pointed out that the size of vesicles depends on the topology of the substrate [6]. They suggested that bilayered phospholipid flakes (BPF) peel off from the substrate and close the edge to form a vesicle. However, in the case of natural swelling, the substrates have no characteristic topology, and the mechanism should not be the same. In addition, the stacking structure and morphology of dry phospholipid films, which might affect the GV formation, were not investigated in detail.

Recently, we observed the $\mu$ m-scale surface morphology of dry phospholipid films by phase-contrast microscopy and atomic force microscopy (AFM) [11]. The dry film was composed of multi-stacked membranes, which were prepared by a process comparable to the natural swelling method. We found that the surface morphology depends on the phases of the phospholipid and that the morphology is related to the ability to form GVs: In the liquid-crystalline phase the dry film exhibits a well-aligned step morphology and GVs are obtained effectively, while in the gel phase the dry film has a rough surface and very few GVs are formed. These results pointed out the importance of investigating the structure of dry phospholipid films in detail for clarifying the mechanism of GV formation.

X-ray reflectivity (XR) and neutron reflectivity (NR) measurements have been performed to shed light on the stacking structure of phospholipid membranes [15-18]. However, most previous studies did not aim at clarifying the mechanism of GV formation and did not consider changes in the $\mu \mathrm{m}$-scale morphology of dry phospholipid films. Therefore, in this study, we investigated the stacking structures of dry phospholipid films on a solid substrate with particular attention to the $\mu$ m-scale morphologies and the ability to form GVs, by 
using XR and AFM measurements in a complementary manner.

\section{Materials and methods}

\subsection{Chemicals}

1,2-Dioleoyl-sn-glycero-3-phosphocholine (DOPC) was obtained in powder form from SIGMA-ALDRICH, and 1,2-dipalmitoyl-sn-glycero-3-phosphocholine (DPPC) was also obtained in powder form from WAKO PURE CHEMICAL INDUSTRIES. The lipids were used without further purification. As a mother solution, these lipids were dissolved in an organic solvent composed of dehydrated chloroform and dehydrated methanol $(2: 1 \mathrm{v} / \mathrm{v}$ ) (both of which were from NACALAI TESQUE). For complete dehydration, molecular sieves (NACALAI TESQUE) were mixed in the solution. The mother solution $(10 \mathrm{mM})$ was stored at $-30{ }^{\circ} \mathrm{C}$ and used for all of the experiments after being allowed to stand at room temperature for five minutes.

\subsection{Dry phospholipid films}

Glass plates $(76 \times 52 \mathrm{~mm}$ for XR or $30 \times 40 \mathrm{~mm}$ for AFM, both from MATSUNAMI GLASS) were treated with acetone. The mother solution $(1.155 \mathrm{~mL}$ for X-ray reflectivity or $10 \mu \mathrm{L}$ for AFM) was dropped onto the glass plate, and the solvent was evaporated without agitation in air (40-50\% humidity) at room temperature $\left(22 \pm 2{ }^{\circ} \mathrm{C}\right)$ for over 3 hours. The samples were placed under vacuum over 2 hours for a complete removal of the solvent. With samples for $\mathrm{XR}$, dry phospholipid films covered the entire area of the glass plate, while a 5-7 mm-diameter spot of dry film remained on the glass plate with the sample for AFM. After this procedure, about a hundred bilayers were expected to be stacked for both samples.

\section{$2.3 \quad X$-ray reflectivity (XR) measurement}

XR measurements were performed with a RINT-TTR-MA (Rigaku Corp.). The wavelength of the X-ray was $1.5406 \AA\left(\mathrm{CuK}_{\alpha 1}\right)$ and the reflected X-ray beam was detected by a scintillation counter [19]. All the measurements were performed at a scattering angle from $0.00^{\circ}$ to $4.00^{\circ}$ with $0.01^{\circ}$ steps for a smallangle range $\left(0-0.6^{\circ}\right)$ and with $0.02^{\circ}$ steps for a large-angle range $\left(0.5-4^{\circ}\right)$. The corresponding overall q range was from 0 to $0.56 \AA^{-1}$. The data obtained were 
corrected for background and then analyzed using a scientific data analysis program provided by Rigaku.

\subsection{Atomic force microscopy (AFM)}

The surface morphology of the dry phospholipid films on glass plates was investigated by AFM (NVB-100, Olympus) with the tapping mode. The images were analyzed and captured with a Nanoscope IIIa system (Digital Instruments). For modification, the images were flattened and plane-fitted to remove inclination of the glass plates. All experiments were carried out at room temperature $\left(22 \pm 2{ }^{\circ} \mathrm{C}\right)$.

\section{Results}

Figure 1 shows the results of XR profiles of dry DOPC and DPPC films, both of which were prepared at room temperature in air. It is clear that Bragg peaks due to the lamellar structure exist up to 4 th order. This means that the lipid bilayers stack regularly in both the dry films. Based on the positions of the peaks, the lamellar repeat distance of the DOPC film is $45.9 \AA$ and that of the DPPC film is $58.2 \AA$. The repeat distance of the DOPC film was almost the same as the thickness of the DOPC bilayer in an aqueous solution in the liquid-crystalline phase [20]. However, that of the DPPC film was $38 \%$ larger than the reported value of the thickness in the gel phase [20]. The full width at half maximum (FWHM) of the first Bragg peak of DPPC is 1.2 times broader than that of DOPC, indicating that the inter-layer structure of DPPC film is more disordered. This tendency is more distinct in the 4th Bragg peak; the height of the peak of DPPC is about $21 \%$ of that of DOPC, and the FWHM of DPPC is about 1.6 times larger than that of DOPC. We tried to analyze the XR profiles by the standard model fitting given by Parratt [21] (see the solid lines in Fig. 1). The number of stacked bilayers in the DOPC film and that of DPPC are found to be 94 and 75 by the fitting, and then the total thicknesses are about $430 \mathrm{~nm}$ and 440nm, respectively. Further, the roughness of the boundary is estimated as a few $\AA$ for the DOPC bilayers, while about $100 \AA$ for the DPPC.

The dry films of DOPC and DPPC were also investigated by AFM (see Fig.2 in ref[11]). The surface of the DOPC film was smooth and flat, and it exhibited a multi-layered terrace-like morphology. The step height of each terrace was about $45.8 \AA$, corresponding to the thickness of the DOPC bilayer in the liquidcrystalline $L_{\alpha}$ phase [20], which is consistent with the value obtained by XR. In contrast, the dry DPPC film showed a very rough surface morphology and 
(a)

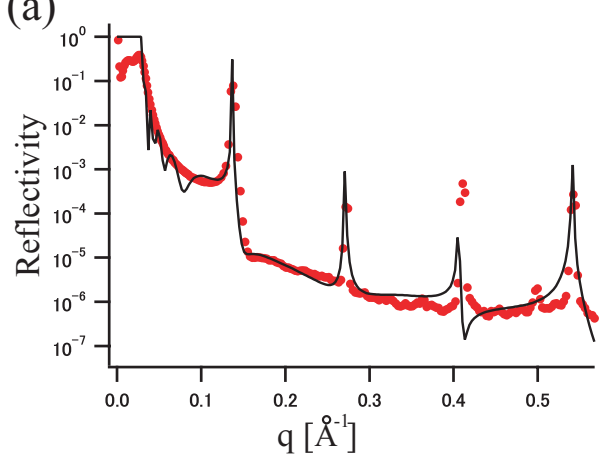

(b)

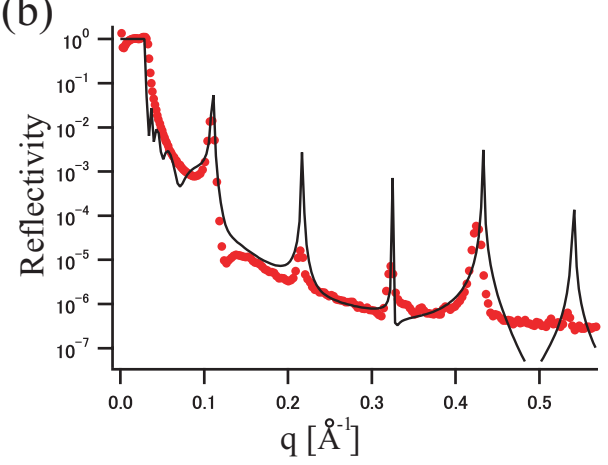

Fig. 1. The observed XR profiles of the dry phospholipid films $(\bullet)$ and best fit to the data (solid line). (a) Dry DOPC film prepared at room temperature. The first Bragg peak locates at about $0.137 \AA^{-1}$. The fitted line is corresponding to the film with 94 bilayers. (b) Dry DPPC film. The first Bragg peak locates at about 0.108 $\AA^{-1} .75$ bilayers are stacked in the film as estimated by the fitting.

no clear step was observed.

\section{Discussion}

By the complementary use of XR and AFM measurements, the structures of multi-stacked DOPC and DPPC membranes (dry DOPC and DPPC film) were clarified. In the case of $\mathrm{DOPC}$, the observed XR profile indicated that the repeat distance between membranes was $45.9 \AA$, which is comparable to the step height $(45.8 \AA)$ observed by AFM. In addition, these values are consistent with the thickness of a DOPC bilayer in the liquid-crystalline phase [20] (DOPC is known to be in a disordered liquid-crystalline $L_{\alpha}$ phase at room temperature in air [23]). The model fitting of XR data shows that the roughness of the stacking of bilayers is a few $\AA$, which is smaller enough than the thickness of bilayer. Taking these results into account, the stacking morphology of DOPC is shown schematically in Fig. 2 (a). About one hundred DOPC bilayers are regularly stacked without a gap between them and form a terracelike morphology. The present results show that disordered phospholipids in the molecular scale exhibits smooth, ordered layering at a $\mu \mathrm{m}$-scale.

On the other hand, the dry DPPC film had a greatly different structure. Despite its rough surface morphology observed by AFM, the existence of sharp higher-order Bragg peaks indicates that DPPC bilayers stacked regularly on a solid substrate. The repeat distance obtained by XR was $\approx 58.2 \AA$, which is much larger than the thickness of a DPPC bilayer $(\approx 42 \AA$ in the ordered gel $L_{\beta}{ }^{\prime}$ phase at room temperature in air $\left.[22,23]\right)$. This difference can be explained by the results of AFM (see Fig. 2 (b)): Steric repulsion between membranes results in the undulating rough morphology of each DPPC membrane. Eventu- 
(a)

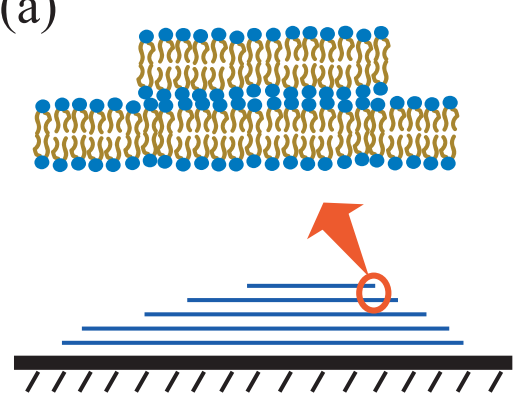

(b)

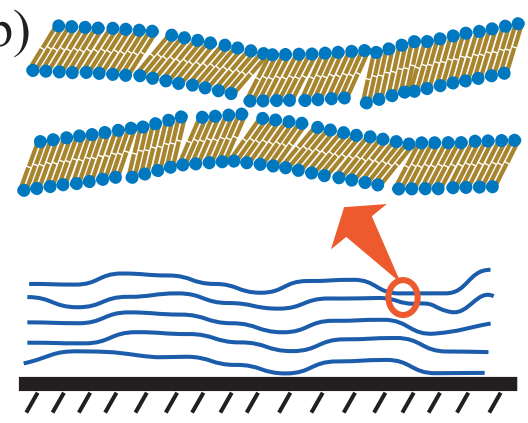

Fig. 2. Morphologies of dry phospholipid films suggested by X-ray reflectivity and AFM. (a) Dry DOPC film. Bilayer membranes form regularly stacked terrace-morphology in the liquid-crystalline phase. (b) Dry DPPC film. In the gel phase, membranes have a rough morphology at a $\mu \mathrm{m}$-scale and stack with a gap due to steric repulsion.

ally, the repeat distance becomes larger than the thickness of the lipid bilayers. This interpretation is consistent with the FWHM of the Bragg peak of the dry DPPC film, which is 1.2 times larger than that of DOPC. The fitting result supports this interpretation, in which the roughness of bilayer stacking is $\sim$ $100 \AA$. This value is consistent with the roughness of surface observed by AFM. However, the micrometer-scale roughness in the film is not suitable to be analyzed by the Parratt algorithm, this could be the origin of the disagreement between the observed XR profiles and the fitted functions. On the other hand, the roughness of the DOPC dry film is not large, and the profile is well fitted by the Parratt algorithm.

These differences in the stacking structures and surface morphologies between DOPC and DPPC depend on the phases of the phospholipid bilayer [11]. We have found that the terrace-like morphology in the liquid-crystalline phase is generated even in a saturated lipid (1,2-Didecanoyl-PC, C 10:0, which is in the liquid-crystalline phase at room temperature). In addition, after the DPPC film is heated above the main transition temperature and annealed at the liquid-crystalline phase, the terrace-like morphology is formed even at room temperature [11]. As we have shown before, the morphology of dry phospholipid film has close relation to the ability to form GVs; the dry phospholipid film with a smooth and step morphology in the liquid-crystalline phase can swell to form GVs effectively, while no GV is formed from the film with rough surface [11]. The present results have clarified the difference in the stacking structures of dry phospholipid films depending on the phases in microscopic scale. Therefore we can conclude that the well ordered stacking of lipid bilayers is important for the $\mathrm{GV}$ formation. 


\section{Conclusion}

We clarified the stacking structures of dry DOPC and DPPC films on a solid substrate, as well as their surface morphologies. Differences in the stacking structure and surface morphology originate from the phases of lipid molecules when a dry film is prepared. In the liquid-crystalline phase, the lipid bilayers stack regularly and a terrace-like morphology is formed. On the other hand, in the gel phase, the lipid bilayers undulate and a rough stacking morphology is generated. These results confirm that the regular stacking of the lipid bilayers and a terrace-like morphology is important for the effective GV formation.

\section{Acknowledgements}

We thank Dr. S.-i. M. Nomura (Tokyo Medical and Dental University), Dr. N. L. Yamada (High Energy Accelerator Research Organization, Japan), and Mr. T. Hamada (Kyoto University) for their helpful advice. This work was supported in part by a Grant-in-Aid for the 21st century COE (Center for Diversity and Universality in Physics) from the Ministry of Education, Culture, Sports, Science and Technology of Japan and by the Yamada Science Foundation.

\section{References}

[1] H. Hotani, F. Nomura, Y. Suzuki, Curr. Opin. Colloid Interface 4 (1999) 358.

[2] S.-i.M. Nomura, K. Tsumoto, T. Hamada, K. Akiyoshi, Y. Nakatani, K. Yoshikawa, ChemBioChem 4 (2003) 1172.

[3] P.-A. Monnard, J. Membr. Biol. 191 (2003) 87.

[4] A. D. Bangham, M.M. Standish, J.C. Watkins, J. Mol. Biol. 13 (1965) 238

[5] J.P. Reeves, R.M. Dowben, J. Cell. Physiol 73 (1969) 49.

[6] D.D. Lasic, J. Colloid Interfac. Sci. 124 (1988) 428.

[7] D.D. Lasic, Biochem. J. 256 (1988) 1.

[8] R.M. Watwe, J.R. Bellare, Current Science 68 (1995) 715.

[9] M.I. Angelova, D.S. Dimitrov, Mol. Cryst. Liq. Cryst. 152 (1987) 89.

[10] S. Kim, R. E. Jacobs, S.H. White, Biochim. Biophys. Acta 812 (1985) 793.

[11] M. Hishida, H. Seto, K. Yoshikawa, Chem. Phys. Lett. 411 (2005) 267. 
[12] D.M. Small, M.C. Bourgès, D.G. Dervichian, Biochim. Biophys. Acta 125 (1966) 563.

[13] A. Saupe, J. Colloid Interface Sci. 58 (1977) 549.

[14] W. Harbich, W. Helfrich, Chem. Phys. Lipids 36 (1984) 39.

[15] C. Münster, T. Salditt, M. Vogel, R. Siebrecht, J. Peisl, Europhys. Lett. 46 (1999) 486.

[16] M. Vogel, C. Münster, W. Fenzl, T. Salditt, Phys. Rev. Lett. 84 (2000) 390.

[17] G. Pabst, J. Katsaras, V.A. Raghunathan, Phys. Rev. Lett. 88 (2002) 128101.

[18] T. Salditt, J. Phys.: Condens. Matter 17 (2005) R287.

[19] H. Matsuoka, E. Mouri, K. Matsumoto, Rigaku J. 18 (2001) 54.

[20] J.F. Nagle, S. Tristram-Nagle, Biochim. Biophys. Acta 1469 (2000) 159.

[21] L.G. Parratt, Phys. Rev. 95 (1954) 359.

[22] R.V. McDaniel, T.J. McIntosh, S.A. Simon, Biochim. Biophys. Acta 731 (1983) 97.

[23] K.L. Koster, Y.P. Lei, M. Anderson, S. Martin, G. Bryant, Biophys. J. 78 (2000) 1932. 